de l'évolution vasculaire accélérée à un certain niveau de la racine.

Il y a donc un parallélisme étroit entre les effets de l'accélération quelle que soit sa cause originelle et quelle que soit le membre de la plante oì on l'observe. De mème que dans la partie aérienne d'une plantule l'accélération basifuge a pour résultat de faire apparaitre dans une portion plus jeune une évolution plus avancée, dans notre racine l'accélération provoquée se manifeste aussi par l'apparition dans une portion plus jeune d'une évolution plus avancée. Ici, la cause déterminante de l'accéléràtion est évidemment accidentelle, mais le processus suivi par la différenciation vasculaire est identique à celui qui a été révélé par l’ontogénie.

Il n'est pas nécessaire d'insister d'avantage pour démontrer la réalité des faits sur lesquels nous nous appuyons et sans la connaissance desquels il est désormais impossible de progresser en anatomie végétale.

\title{
Sur les formes de la Lychnide dioïque et sur I'hérédité de la couleur des fleurs dans cette espèce
}

PAR M. L. BLARINGHEM.

La Lychnide dioïque (Lychnis dioica DG.) est définie par la séparation des sexes, d'ordinaire répartis sur des pieds différents. Introduite aux États-Unis, sans doute sous sa forme à fleurs rouges, elle s'y est répandue et on y trouye des pieds à fleurs blanches, des pieds à fleurs roses et des pieds à fleurs rouges. D'après G. H. Shull $(1908,1910)$, on y rencontre aussi, d'ailleurs assez rarement, des plantes hermaphrodites et cet auteur a isolé une lignée, qu'il appelle mutante, possédant régulièrement ce caractère par hérédité; au point de vue génétique, elle se comporterait dans les croisements comme le type mâle.

En Europe et plus spécialement en France, les pieds hermaphrodites, bien que signalés à diverses reprises, même par Linné, sont rapportés, depuis les belles recherches de A. Magnin, 
L. BLARINGHEM. - SUR LES FORMES DE LA LYCHNIDE DIOÏQUE.

de M. Cornu et de A. Giard, à des individus monstrueux atteints par l'Ustilago antherarum; les pieds mâles fournissent des pseudo-ovaires toujours stériles au centre de la couronne pédicellée des étamines, dont les anthères éclatent sous la pression des milliers de spores du champignon parasite; les pieds femelles présentent, eux aussi, des étamines parfaitement développées dont les anthères, très grosses, sont le siège du développément des spores de l'Ustilago. Le plus souvent toutes les fleurs d'une même plante malade sont stériles; mais j'ai trouvé, au cours de deux étés, environ 12 plantes sur plus de 500 partiellement atteintes en ce sens que des pousses, développées manifestement sur la souche portant des fleurs malades, donnent naissance à des ovaires normaux et d'ailleurs fertiles. En ce cas les fleurs saines sont unisexuées comme le type normal.

A. Giard et Magnin ${ }^{1}$ considèrent l'hermaphroditisme accidentel du Lychnis dioica comme la conséquence directe et constante de la présence du parasite: Strasburger (1910) adopte ce point de vue, discutant l'aftirmation, sans doute valable pour les États-Unis, de Shull qui a possédé une lignée franchement hermaphrodite, l'a obtenue à plusieurs reprises et l'a cultivée durant des années. Pour ma part, n'ayant fait des observations sur cette plante que dans le Centre et le Nord de la France, bien que j'aie à dessein cherché des plantes hermaphrodites, je les ai toujours trouvées atteintes par l'Ustilago, sauf dans un individu hybride observé ce printemps, issu à la deuxième génération du croisement Lychnis vespertina $\mathrm{Sib} . \times$ Melandriumrubrum Rœhl.; il ne portait d'ailleurs que des fleurs à 1 ou 2 staminodes, alors que la plupart des fleurs étaient strictement femelles. Je ne parlerai pas davantage ici de cette aberration dont je me propose de suivre la descendance.

Mais précisément parce que j'ai entrepris des cultures expérimentales avec cette espèce polymorphe, j'ai éprouvé de réelles

1. Magnin (A.), Recherches sur le polymorphisme floral, la sexualité et Chermaphroditisme parasitaire du Lychnis vespertina Sibth. (Ann. Soc. bot. de Lyon, 1889,32 p. et 2 pl.). 
difficultés à en délimiter les formes et si je donne cette courte analyse de mes observations, c'est pour engager mes collègues à faire eux aussi une étude de cette espèce et, si l'occasion s'en présente, à me communiquer des plants ou des graines de lignées types.

Laissant de côté l'espèce méditerranéenne L. macrocarpa Boissier et la forme localisée en Corse L. Requienii Rohrb., je suis amené à grouper sous trois noms les plantes bisannuelles ou vivaces du groupe. Le $L$. noctiflora Fries, annuel, doit être provisoirement mis à part surtout à cause du nombre des stigmates réduit à 3. Dans les Lychnides étudiées le nombre des stigmates fut toujours supérieur à 4 , dans la majorité des cas 5 , souvent 6 et exceptionnellement sur quelques fleurs 8 .

Dans le groupe $L$. dioica DC. je distingue :

A. - L. vespertina Sibthorp, ou Compagnon blanc, espèce polymorphe à grande aire d'extension et très commune dans les stations où je l'ai étudiée (Meudon, Angers, Veules, Béthune). Les fleurs sont grandes et s'ouvrent surtout le soir; les feuilles, les ramifications et les calices sont couverts de poils en partie glanduleux; les capsules sessiles, s'ouvrant par dix dents dressées, grosses mais piriformes, sont caractéristiques. Les fleurs sont toujours blanches ou très légè rement teintées de jaune, ou même de rose très pâle lorsqu'elles sont sur le point de se flétrir. La floraison est de longue durée, de la mi-mai à fin septembre, et souvent dure deux années lorsque la plante est vigoureuse et peut s'étaler en buisson.

B. - L. dioica var. coloratum Rostr. que je considère comme équivalent à Melandrium pratense var. incarnatum Lamotte. Le Muséum d'Histoire naturelle de Paris en possédait une plante mâle en 1920, sous la désignation Melandrium rubrum et j'ai trouvé cette forme en juillet 1920 entre Varangeville et Ouville (Seine-Inférieure), en pleine floraison, dans une station où les L. vespertina Sibth. paraissaient absents. En fait, à part la coloration rose vif des pétales qui sont très grands et étalés le matin et la teinte foncée des axes, peut-être aussi les divisions dichotomiques très grêles et écartées des axes florifères, il n'y aurait pas de raison valable de la séparer du type précédent. C'est d'ailleurs l'opinion courante en France. Godron, Coste 
en font des variations du $L$. vespertina ou Compagnon blanc type; Rouy et Foucauld (Flore de France, 1896, III, p. 95) la décrivent sommairement comme sous-variété de l'espèce précédente.

C. - L. silvestre Rchl., qui est le L. diurna Sibthorp. Cette espèce a des fleurs franchement rouges. Je l'ai étudiée en 1920 sur l'exemplaire vivant de la collection du Muséum d'Histoire naturelle de Paris, puis dans le Pas-de-Calais dans les marais de Beuvry. Récemment (28 mai 1922) j'en ai analysé deux stations dont l'une abondante à Solres-le-Château, près Jeumont, à la frontière franco-belge. Je considère ces dernières plantes comme typiques, parce que je n'ai pu découvrir dans cette région, parcourue sur plusieurs kilomètres en tous sens, aucune plante Lychnis vespertina. Les feuilles sont larges et minces, pétiolées à la base, modérément velues; les axes dressés sont peu étalés mais fort élevés; la pubescence courte qui couvre tous les organes n'est pas glanduleuse; les fleurs sont petites, leur plus grand diamètre ne dépassant pas 16 millimètres, alors que celles du vespertina dépassent presque toujours 20 millimètres; les capsules sont petites et rondes, les dix dents des valves roulées en dehors (pas vu encore à Solres).

Mais j’insiste peu sur les caractères des capsules qui sont cependant considérés par la majorité des auteurs comme essentiels. A Veules (Seine-Inférieure), sur une étendue de un kilomètre carré de la falaise, j'ai récolté en juillet 1920 et en août 1921 plus de 30 formes de fruits différant par la taille, la forme, les dimensions de l'ouverture, les dimensions et la courbure des dents. Sans doute la fécondation croisée nécessaire intervient pour partie dans ce polymorphisme, mais il serait dangereux d'accorder une valeur décisive à pareil caractère qui entraînerait le morcellement indéfini du type vespertina. Les variations de compacité des grappes dichotomes ne m'ont pas fourni de meilleurs renseignements.

- Les variations dans la pubescence, le port dressé, l'étalement des feuilles corrélatif de leur faible épaissear, permettent de séparer nettement $L$. silvestre de $L$. vespertina. Mais ces caractères ne sont-ils pas liés à la station humide, fréquemment ombragée où se plaît la première espèce? Somme toute, la 
taille et la couleur des fleurs restent les caractères essentiels et distinctifs des formes $\mathrm{A}$ et $\mathrm{C}$.

Des croisements, faits en 1920 et depuis pour l'étude de l'hérédité de la sexualité ${ }^{1}$, peuvent être utilisés pour discuter la valeur relative des types B et C par rapport à l'espèce commune A. La comparaison des caractères morphologiques de A et $B$ me conduisait à admettre pour fondée l'opinion courante que $\mathbf{B}$ était une simple variation de $\mathbf{A}$, dont elle ne diffère que par la couleur rose des fleurs. En génétique, les variétés distinctes de l'espèce par un seul caractère, indépendant des autres particularités, sont désignées sous le nom de variétés régressives ${ }^{2}$ et leurs croisements donnent des descendances qui suivent les règles numériques de la disjonction mendélienne. Le type C, admis comme bonne espèce, au moins comme une espèce élémentaire, devait selon les mêmes règles se comporter différemment et j'espérais que les croisements, faciles à réaliser, donneraient une solution décisive à la question.

En 1920, une même plante femelle $L$. vespertina, de Meudon, fut fécondée successivement avec le pollen de Lychnis silvestre du Muséum, de Lychnis viscosa de même origine, du Lychnis dioica var. coloratum. de Varangeville et du L. vespertina de Meudon. Toutes les plantes, à peu d'exceptions près, furent femelles, mais l'avortement total des fruits fécondés par Lychnis viscosa et les différentes colorations notées dans la descendance me prouvèrent que la plante femelle $L$. vespertina, utilisée pour les essais, n'était pas capable de fournir des graines par parthénogenèse. Les premières plantes en fleurs de 1921 furent fécondées avec le pollen de trois individus mâles développés à l'endroit même où se trouvait l'année précédente le $L$. vespertina mère. Eux aussi fournissent un certain pourcentage de plantes colorées. Les tableaux suivants résument les dénombrements :

1. Blaringhem (L.), Sur l'hérédité du sexe chez la Lychnide diö̈que (Lychnis vespertina Sibthorp). (C. R. Acad. Sciences, Paris, CLXXIV, p.1429, 1922).

2. Blaringhem (L.), Les problèmes de l'hérédité expérimentale. Paris, Flammarion, 1919 , ch. VII. 


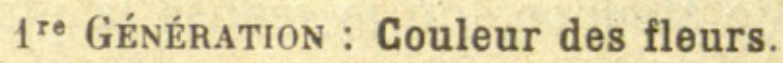

\begin{tabular}{|c|c|c|c|c|c|c|}
\hline & Lot & 우난 & & blanche & rosé & rouge \\
\hline L. vespertina & silv & 114 & & 69 & 27 & 19 \\
\hline- & $\times$ coloratum. & 72 & 3 & 46 & 12 & 17 \\
\hline - & $\times$ vespertina & 1 & 4 & 75 & 0 & \\
\hline
\end{tabular}

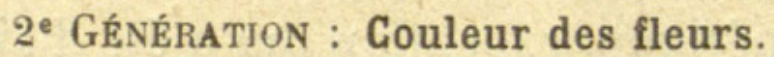

Ascendant rouge :

4. (L. vespertina $\times$ silvestre $) \times$ vespertina.

5 .

6.

7. (L. vespertina $\times$ coloratum $) \times$ vespertina.
+ 8 blanche rosé rouge

$\begin{array}{lllll}89 & 60 & 78 & 49 & 22\end{array}$

$\begin{array}{lllll}64 & 33 & 68 & 26 & 3\end{array}$

$\begin{array}{lllll}26 & 39 & 54 & 11 & 0\end{array}$

$\begin{array}{lllll}39 & 24 & 34 & 20 & 9\end{array}$

$\begin{array}{lllll}37 & 68 & 45 & 23 & 37\end{array}$

Il se dégage une règle simple de cet exposé. Toutes les fois que les ascendants sont à fleurs blanches il n'y a dans les descendances, même à la seconde génération, que des plantes à fleurs blanches. Cinq lignées des plantes à fleurs blanches des croisements 1 et 2 ont été suivies sur $69,68,93,91$ et 103 individus, et je n'ai trouvé aucune exception à la règle, bien que la chute abondante des fleurs, très marquée sur certains individus, indiquât des altérations physiologiques qui ne sont pas dues exclusivement à l'élimination volontaire des mâles. On pourrait admettre que la couleur blanche, récessive, est épurée.

Mais le rouge, ou le rosé, qui devraient se comporter comme des dominants simples en première génération, offrent cette particularité de ne pas être des dominants pour l'ensemble des descendants; au contraire, dans les deux croisements, les individus à fleurs blanches constituent la majorité qui est d'ailleurs sensiblement la même pour le croisement par $L$. silvestre et pour le croisement par $L$. coloratum.

Les descendances suivies en deuxième génération donnent des résultats analogues, avec une majorité de plantes à fleurs colorées dans la lignée 8. Cette anomalie est d'autant plus remarquable que le pollen utilisé pour cette seconde génération provenait de plantes qui ont eu des ascendants à fleurs blanches depuis au moins dix générations, puisque tout individu à fleurs roses n'aurait pu échapper à mon attention dans les pelouses 
du laboratoire de Meudon, où je réalise la plus grande partie de mes cultures expérimentales.

Il faut donc retenir de cet essai que les disjonctions par le croisement des teintes des fleurs ne permettent pas de distinguer $L$. silvestre du $L$. dioica var. coloratum. C'est une exception troublante à la règle habituelle de la dominance et de la ségrégation numérique, exception qui a été d'ailleurs constatée par Shull $(1908,1910)$ bien qu'il l'interprète d'une toute autre façon ${ }^{1}$.

Cet auteur admet en effet que les Lychnides dioïques, ne pouvant être obtenues en lignée pure en raison de la séparation des sexes, se comportent comme des hétérozygotes à divers degrés, certaines lignées donnant des proportions voisines de 100 pour 100 rouges, la même quantité 0 p. 100, tandis qu'un nombre égal à la somme des deux catégories précédentes fournit 50 pour 100 de rouges, 50 pour 100 de blancs. Ses épreuves, portant sur une cinquantaine de lignées, paraissent probantes et il en conclut que le rouge en définitive domine le blanc. En fait, Shull a étudié plus spécialement les descendances de lignées rouges et il se place dans un cas très différent de celui qui est exposé ici où les blanes jouent le rôle essentiel. D'autre part, il trouve des différences fondamentales selon les lignées examinées et je suis parti d'une seule plante femelle. Enfin, il admet la présence de plusieurs facteurs pour expliquer le virage du blane au rouge. Toutes ces divergences indiquent que le problème est très complexe et ne pourra être élucidé qu'avec certaines lignées bien choisies.

Il serait nécessaire d'avoir des plants ou des graines des divers Lychnides dont il est question ici, présentant dans leurs stations une très grande homogénéité; par exemple des Lychnis silvestre récoltés dans des localités où n'existe pas Lychnis vespertina, ni sa forme coloratum; je crois en posséder un lot

1. SHULl (G. H.), Some new cases of mendelian inheritance. (Bot. Gazette, XLV, p. 103-116, 1908) et Color inheritance in Lychnis dioica $L$. (The American Naturalist, XEIV, p. 83-91, 1910). 
E. DECROCK. - LE GARDAMINE PLUMIERI VHL. EN PROVENCE.

provenant de Solres-le-Château; mais il serait préférable que le matériel fùt récolté par un collègue étudiant la flore de sa localité depuis de nombreuses années et pouvant s'assurer, par un contrôle sur place, qu'il n'y a pas eu de chance de contamination par les introductions de graines fourragères ou autres accidents imprévus.

\section{Le Gardamine Plumieri Vill. dans les Alpes de Provence}

PAR M. E. DECROCK.

Notre confrère M. Jules Offner, de Grenoble, a publié il y a quelques années une note fort intéressante sur la distribution du Cardamine Plumieri Vill ${ }^{1}$. D'après lui l'aire de cette Crucifère s'étend dans les Alpes françaises : $1^{\circ}$ sur tout le secteur granitique des Alpes delphino-savoisiennes où elle est surtout répandue, $2^{\circ}$ dans le secteur des Alpes austro-occidentales sur le district des Alpes Grées et sur le district des Alpes Cottiennes.

Le district des Alpes de Provence semblait donc exclu du domaine de cette espèce, attendu que le bois de Loubet, près de Rabou, dans la partie sud du Dévoluy paraissait être la localité la plus méridionale où la plante en question avait été observée dans la France continentale.

Or, au cours de nos recherches sur la végétation de la haute vallée du Verdon nous avons récemment trouvé la Cardamine de Plumier au voisinage du lac d'Allos, plus exactement au Laus, sur des banes de grès d'Annot, à l'altitude de 2131 mètres environ.

La limite méridionale admise jusqu'ici pour cette espèce dans les Alpes de France est donc à reporter notablement vers le Sud et le district des Alpes de Provence s'enrichit d'une unité remarquable.

1. Offaner (J.), Distribution gèographique du Cardamine Plumieri Vill. dans les Alpes françaises (Bull. Soc. bot. France, XX, 1920, p. 134). 


\section{$2 \mathrm{BHL}$ Biodiversity Heritage Library}

Blaringhem, Louis. 1922. "Sur les formes de la Lychnide dioïque et sur I'hérédité de la couleur des fleurs dans cette espèce." Bulletin de la Société botanique de France 69, 340-347. https://doi.org/10.1080/00378941.1922.10833450.

View This Item Online: $\underline{\text { https://www.biodiversitylibrary.org/item/93160 }}$

DOI: https://doi.org/10.1080/00378941.1922.10833450

Permalink: https://www.biodiversitylibrary.org/partpdf/161269

\section{Holding Institution}

Missouri Botanical Garden, Peter H. Raven Library

\section{Sponsored by}

Missouri Botanical Garden

\section{Copyright \& Reuse}

Copyright Status: Public domain. The BHL considers that this work is no longer under copyright protection.

This document was created from content at the Biodiversity Heritage Library, the world's largest open access digital library for biodiversity literature and archives. Visit BHL at https://www.biodiversitylibrary.org. 